\section{MS12 P01}

Synthesis and structure of a new HO(III) coordination polymer exhibiting 3D open framework ${ }^{(1)}$ Rahahlia N. , ${ }^{(1)}$ Aliouane K., ${ }^{(1)}$ Guehria-Laidoudi A., ${ }^{(2)}$ Dahaoui S. and ${ }^{(2)}$ Lecomte C. ${ }^{(1)}$ Laboratoire de CristallographieThermodynamique, Faculté de Chimie, USTHB, BP 32 El-Alia, Bab Ezzouar 16111, Alger ; guehria laidoudi@yahoo.fr ${ }^{(2)}$ LCM $^{3}$ B-CNRS UMR 7036, Faculté des Sciences Université Henri Poincaré BP 239, 54506 Vandoeuvre-lès-Nancy, France.

\section{Keywords : coordination polymer, MOFs, rare-earth}

$\alpha, \omega$ - Dicarboxylic acids give flexible ambidentate and templating ligands leading to coordination polymers having a wide breadth of applications. With lanthanide cations, known for their oxygen affinity and their great range of coordination numbers and modes, the resulting self-assembly networks have enhanced the field of supramolecular chemistry from both a theoretical and practical point of view. In an attempt to understand the significance of specific interactions in hybrid materials based on lanthanide elements we have synthesized, under hydrothermal conditions and determined the first crystal structure of an Holmium fumarate.

The fumarate of Holmium is isostructural with Erbium and Europium analogues [1-3]. Except for Pm, and Tm , the single crystal structures of all other lanthanide fumarates have been obtained, reflecting the increased activity in the field of metal-organic frameworks involving rare-earth elements. We present here the synthesis and structure of the first Ho fumarate.

The asymmetric unit contains two Ho atoms, three fumarate anions, four coordinated and three uncoordinated waters molecules. Two Ho (III) atoms are linked through two $\mu 1,1$ and one $\mu 1$,3-oxo-bridges forming the basic binuclear unit. Hence, the Ho (III) atoms are nine-coordinated by seven $\mathrm{O}$ atoms from fumarate ligands, and two from aqua ligands. The coordination polyhedra are a slightly distorted tricapped trigonal prism. The three twisted fumarate ligands are in different coordination modes: two are pentadentate exhibiting the same bridging-chelating mode by one function and a conventional carboxylate bridge or a chelate by the second function. The third ligand is tetradentate showing a chelate mode and a conventional carboxylate bridge.

The connection of the edge-sharing bi-prism through the ligands as spacers along [100] and [010] directions, leads to $3 \mathrm{D}$ open framework with small channels.

We are still focusing on the investigation of the thermal and the photoluminescent properties induced by the connectors which have $\mathrm{f}$ electrons and by the linkers which display relative rigidity and multiple bond interactions in this coordination polymer.

[1] G.Zhang, Q. Wang, Y. Yang, J.S. Ma, J.of Mol.Struct.,796, 187-194, 2006.

[2] A. Michaelides, S. Skoulika, E.G. Bakalbassis, J. Mrozinski, Cryst.Growth Des.,3,487-492,2003.

[3] X.Li,Y.Q.Zou, J.of Chem.Cryst., 35,5,351-355, 2005.

\section{MS12 P02}

The structural characteristics of the butane-1,4diammonium dihalide salts. Charmaine van Blerk and Gert Kruger, Department of Chemistry, University of Johannesburg, Johannesburg, 2006, South Africa. E-mail: cvanblerk@uj.ac.za
Keywords: crystal and molecular structure, singlecrystal X-ray analysis, isostructurality.

An investigation of the structural characteristics of the $n$ alkyl diammonium salts is important as they have important industrial applications. The current study forms part of a project investigating the polymorphism and structural features of alkyl diammonium salts with particular focus on the halide $\left(\mathrm{Cl}^{-} ; \mathrm{Br}^{-}\right.$and $\left.\mathrm{I}^{-}\right)$salts of 1,4diaminobutane (also known as putrescine). The crystal structure of butane-1,4-diammonium dichloride was published 27 years ago [1] but during our comprehensive study of the whole group of compounds, it was improved by a redetermination using modern instrumentation. The crystal structures of butane-1,4-diammonium dibromide and butane-1,4-diammonium diiodide were published recently $[2,3]$. These compounds were found to be isostructural as all three crystallized with similar cell dimensions; they have the same space group $\left(P 2_{1} / c\right)$; the cations all sit on a centre of inversion and therefore each asymmetric unit contains one-half of the cation together with one halide anion; all exhibit the same packing arrangement of ionic layers separated by hydrocarbon layers in which the linearly extended chains pack in a stacked herringbone manner; and all show identical, infinitely extended hydrogen bonding networks as shown in the figure below.

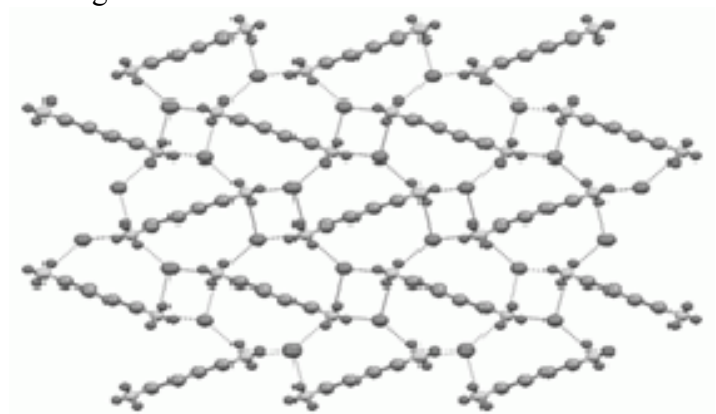

[1] Chandrasekhar, K. \& Pattabhi, V. Acta Cryst., 1980, B36, 2486-2488.

[2] van Blerk, C. \& Kruger, G. J. Acta Cryst., 2007, E63, o342o344.

[3] Lemmerer, A. \& Billing, D. G., Acta Cryst., 2006, E62, o1954-o1956.

\section{MS12 P03 \\ Characterisation of Molecular organisation and thermal properties of lipidic nanostructures applied to pharmaceutical, cosmetic and food products. Michel Ollivon $^{\mathrm{a}}$, Claudie Bourgaux ${ }^{\mathrm{a}}$, Gérard Keller ${ }^{\mathrm{a}}$, Daniel Kalnin $^{a}$, Angelina Angelova a ${ }^{a}$ Physics and Chemistry of Polyphased Systems, University of Paris-Sud, CNRS- UMR8612, Châtenay-Mal., France. \\ E-mail: michel.ollivon@u-psud.fr}

\section{Keywords: Lipids, DSC, Nanostructures}

Molecules bearing one or several hydrocarbon chains display partial or total insolubility in water and self aggregation properties that are frequently used in pharmaceutical, chemical, food and cosmetic applications to generate lipidic nanoparticles, emulsions, liposomes, cubosomes or hexosomes as well as mesophases, microemulsions or micellar solutions. The self aggregation properties and the type of structures formed by these lipidic molecules whatever their origins, biologic or synthetic, depend on the nature of the polar head groups 